\title{
Compact Quotients of Large Domains in a Complex Projective 3-space
}

\author{
Masahide KATO
}

Sophia University

\begin{abstract}
In a complex projective 3-space, we consider a domain with a projective line. If there is a compact non-singular quotient of the domain and the quotient manifold admits a non-constant meromorphic function, then the domain is dense in the projective 3-space and its complement is properly contained in a finite union of complex hypersurfaces and a set with Hausdorff dimension not more than two. Further, if the complement admits a certain fiber space structure, then it is either a disjoint union of two projective lines, a projective line, or an empty set.
\end{abstract}

\section{Introduction}

By a line, we shall mean a projective linear subspace of dimension 1 in a 3-dimensional complex projective space $\mathbf{P}^{3}$. A domain $\Omega$ in a projective 3 -space $\mathbf{P}^{3}$ is said to be large* if $\Omega$ contains a line in $\mathbf{P}^{3}$. Let $\Gamma$ be a holomorphic automorphism group of $\Omega$. Suppose that the action of $\Gamma$ is fixed point free and properly discontinuous, and that the quotient space is compact. In this situation, we say that $X$ is a compact quotient of a large domain $\Omega$ by $\Gamma$ and indicated by $X=\Omega / \Gamma$. Under the assumption that $\Omega$ is large, the automorphism group $\Gamma$ appears to be a subgroup of PGL(4) ([K2]). Put $\Lambda=\mathbf{P}^{3} \backslash \Omega$. We denote by $a(M)$ the algebraic dimension of a complex manifold $M$. In general, $\Lambda$ may contain interior points [K4]. In this note, we shall consider the case $a(X)>0$. Without any assumptions on $\Lambda$, we have

THEOREM A. If $a(X)>0$, then $\Lambda=\mathbf{P}^{3} \backslash \Omega$ is contained in $S \cup A$, where $S$ is a finite union of complex hypersurfaces in $\mathbf{P}^{3}$, and A is a closed subset of $\mathbf{P}^{3} \backslash S$ with the Hausdorff dimension $d(A)$ not more than 2. In particular, $\Omega$ is dense in $\mathbf{P}^{3}$.

We say that $\Lambda$ is of fibered type, if $\Lambda$ satisfies the following condition:

(F) $\Lambda$ coincides with the set $\bigcup_{s \in I}\left|\ell_{s}\right|$, where $\left\{\ell_{s}\right\}_{s \in I}$ is a family of lines parametrized by an index set $I$ with an arbitrary cardinality such that $\left|\ell_{s}\right| \cap\left|\ell_{t}\right|=\emptyset$ for any $s \neq t$.

THEOREM B. Suppose that $\Lambda$ is of fibered type. Then, if $a(X)>0$, the cardinality of I is at most 2, and X is biholomorphic to $\mathbf{P}^{3}$, a Blanchard manifold, or an L-Hopf manifold.

Received November 10, 2004

Partly supported by the Grant-in-Aid for Scientific Research (C), Japan Society for the Promotion of Science.

Keywords: extension of holomorphic map, Gauduchon metric, pluri-closed Hermitian metric, non-Kähler manifold. Math. classification: 32H02, 32J17

* We shall use this term after Lárusson [L]. 
Here, $|\ell|$ indicates the support of a line $\ell$. For the definitions of Blanchard manifolds and $L$-Hopf manifolds, see [K3]. See also notes added in proof.

Among compact quotients of large domains are most of flat twistor spaces. Note that the condition $(F)$ is fulfilled for flat twistor spaces over conformally flat differentiable 4manifolds which are conformal quotients of domains in $S^{4}$. The condition $(F)$ is preserved by complex analytic connected sum operations (see, Proposition 5.1). Flat twistor spaces with positive algebraic dimensions are already classified by Fujiki $[\mathrm{F}]$ as an application of his theory on general twistor spaces. Since compact quotients of large domains, however, do not always have flat twistor space structures, we take another approach to our study. We are rather inclined to develop a higher dimensional analogue of Klein group theory.

Our main tool to study the set $\Lambda$ is a deep result of S. Ivashkovich [I] on the extension of meromorphic maps of Hartogs domains into complex manifolds with pluri-closed Hermitian metrics (section 4). The index $\mathrm{Hex}_{n}$ for complex manifolds introduced in [KO] is also useful. Section 1 is for preliminaries. Theorem A will be proved in section 2. Theorem B will be proved in section 3. On the existence of pluri-closed Hermitian metrics on the total space of a family of compact complex surfaces will be discussed in section 4 . In section 5 , we verify that the condition $(F)$ is fulfilled for complex analytic connected sums of flat twistor spaces.

\section{Preliminaries}

The envelope of holomorphy for an $n$-dimensional Hartogs domain over $\mathbf{C}^{n}$ is a polydisk, which we call by the associated polydisk for the Hartogs domain. In [I], S. Ivashkovich proved among other things the following, which is a key of this paper.

THEOREM 1.1 (Ivashkovich). Let $X$ be a disk-convex complex manifold with a pluriclosed Hermitian metric. Then every meromorphic map $f$ of an $n$-dimensional Hartogs domain $H$ into $X$ extends meromorphically to $\Delta \backslash A$, where $\Delta$ is the associated polydisk of $H$, and $A$ is a closed subset of $\Delta$ with the Hausdorff dimension not more that $2 n-4$. Further, if $n=2$ and $X$ is of dimension 2, then $A$ is a set of isolated points in $\Delta$.

In [KO], we have introduced an index $\mathrm{Hex}_{n}$ of a complex manifold, which we call holomorphic extension index, to scale the extendability of holomorphic maps into the manifold. See [KO] for the detail. Theorem 1.1 tells us, in particular, that $\operatorname{Hex}_{n}(X) \geq 4$ holds for a holomorphically convex complex manifold $X$ with a pluri-closed Hermitian metric.

Proposition 1.1. Let $X$ be a complex manifold and $D=\cup_{\lambda} D_{\lambda}$ a finite union of nonsingular hypersurfaces $D_{\lambda} \subset X$. Assume that $X \backslash D$ and any component $D_{\lambda}$ are n-probable and that $\operatorname{Hex}_{n}(X \backslash D)>2$. Then $X$ is n-probable and $\operatorname{Hex}_{n}(X) \geq \min _{\lambda}\left\{\operatorname{Hex}_{n}\left(D_{\lambda}\right), 2\right\}$ holds.

Proof. See [KO, Proposition 4].

PROPOSITION 1.2. Let $X$ be a complex manifold which is n-probable with $\operatorname{Hex}_{n}(X)>$ 2. Let $(\Omega, \pi)$ be an étale domain over a Stein manifold $M$ with $\operatorname{dim}_{\mathbf{C}} M=n$. Then, for every holomorphic map $\sigma: \Omega \rightarrow X$, the maximal domain $\Omega_{\sigma}$ of definition for $\sigma$ over $M$ is a 
subdomain of $\tilde{\Omega}_{\sigma}$ satisfying

$$
d\left(\tilde{\Omega}_{\sigma} \backslash \Omega_{\sigma}\right) \leq 2 n-\operatorname{Hex}_{n}(X),
$$

where $\tilde{\Omega}_{\sigma}$ is the envelope of holomorphy for $\Omega_{\sigma}$ over $M$.

Proof. See [KO, Theorem 2].

PROPOSITION 1.3. Let $X$ and $Y$ are n-probable complex manifolds and $f: X \rightarrow Y$ a surjective holomorphic map. Let $\left\{U_{\alpha}\right\}_{\alpha}$ be any open covering of $Y$. Then we have the following inequality

$$
\operatorname{Hex}_{n}(X) \geq \inf _{\alpha}\left\{\operatorname{Hex}_{n}(Y), \operatorname{Hex}_{n}\left(f^{-1}\left(U_{\alpha}\right)\right)\right\} .
$$

ProOF. See [KO, Theorem 3].

LEMma 1.1. Let $X, Y$ be complex manifolds and $f: X \rightarrow Y$ a holomorphic unramified even covering. If $Y$ is n-probable with $\operatorname{Hex}_{n}(Y)>2$, then $X$ is n-probable and $\operatorname{Hex}_{n}(X)=\operatorname{Hex}_{n}(Y)$ holds.

ProOF. See [KO, Proposition 5(b)].

LemmA 1.2. Let $X$ be an n-probable complex manifolds with $\operatorname{Hex}_{n}(X)>2$ and $D$ a pure dimensional analytic subset of codimension 1 . Then $X \backslash D$ is also n-probable and $\operatorname{Hex}_{n}(X \backslash D) \geq \operatorname{Hex}_{n}(X)$ holds.

Proof. Any subdomain of an $n$-probable manifold is $n$-probable. Hence $X \backslash D$ is $n$ probable. Let $\sigma: H \rightarrow X \backslash D$ be any holomorphic map of a Hartogs domain $H$. Since $X$ is $n$-probable, $\sigma$ extends to a holomorphic map $\hat{\sigma}: \Delta \backslash A \rightarrow X$, where $A$ is a closed subset of the associated polydisk $\Delta$ with $d(A) \leq 2 n-\operatorname{Hex}_{n}(X)<2 n-2$. Put $E=\hat{\sigma}^{-1}(D)$. Then $E$ is an analytic subset of $\Delta \backslash A$ with pure codimension 1, provided that $E \neq \emptyset$. E extends to an analytic subset $\hat{E}$ of $\Delta$. Hence $\hat{E} \cap H \neq \emptyset$. This is absurd, since $\sigma(H) \subset X \backslash D$. Hence $E=\emptyset$ and consequently we have $\hat{\sigma}(D \backslash A) \subset X \backslash D$. Therefore we obtain $\operatorname{Hex}_{n}(X \backslash D)=$ $2 n-\sup _{\sigma} d\left(A_{\sigma}\right) \geq \operatorname{Hex}_{n}(X)$.

THEOREM 1.2. Let $X$ be a complex manifold of dimension 3 and $S=\{t \in \mathbf{C}:|t|<$ 1\}. Suppose that $X$ is n-probable and that there is a proper holomorphic surjective map $f: X \rightarrow S$ with connected fibers which is of maximal rank everywhere. Then $\operatorname{Hex}_{n}(X) \geq 4$ holds.

PROOF. This will be proved in section 4.

Note that $\Omega / \Gamma$ is always $n$-probable, since any holomorphic map of a Hartogs domain into $\Omega / \Gamma$ lifts to $\Omega$ and extends to a meromorphic map of the associated polydisk into $\mathbf{P}^{3}$.

PROPOSITION 1.4. If $a(\Omega / \Gamma)>0$, then $\operatorname{Hex}_{n}(\Omega / \Gamma) \geq 2$.

Proof. Put $X=\Omega / \Gamma$. Let $\mu: X^{*} \rightarrow X$ be a blowing-up of $X$ such that there is a holomorphic map $f: X^{*} \rightarrow C$ onto a compact non-singular curve $C$ with connected fibers. 
There is a finite subset $A$ of $C$ such that $X^{*} \backslash f^{-1}(A)$ is of maximal rank everywhere and that every irreducible component of $f^{-1}(A)$ is non-singular. We have $\operatorname{Hex}_{n}\left(X^{*} \backslash f^{-1}(A)\right) \geq 4$ by Theorem 1.2. By Theorem 1.1, we know that $\operatorname{Hex}_{n} \geq 4$ for any compact complex surfaces. Hence we have $\operatorname{Hex}_{n}\left(X^{*}\right) \geq 2$ by Proposition 1.1. Hence so is $X$.

\section{Proof of Theorem A}

A non-constant meromorphic function $f$ defines a meromorphic map $X \cdots \rightarrow \mathbf{P}^{1}$. Since $\Omega$ is large, $\pi^{*} f$ extends to a $\Gamma$-invariant rational function $F$ on $\mathbf{P}^{3}$. Thus we have a commutative diagram of meromorphic maps

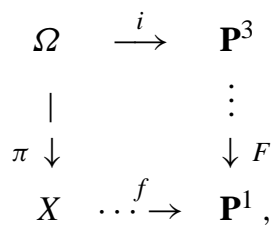

where $i$ is the natural inclusion, and $\pi$ is the canonical projection. We eliminates the base locus of $F$ by successive blowing-ups of $\mathbf{P}^{3}$ to obtain a non-singular 3-manifold $M$ and a bimeromorphic holomorphic map $u: M \rightarrow \mathbf{P}^{3}$. Then $u^{*} F: M \rightarrow \mathbf{P}^{1}$ is holomorphic. Consider the Stein factorization of $u^{*} F$, and we obtain a ramified covering $v: C \rightarrow \mathbf{P}^{1}$ with the commutative diagram

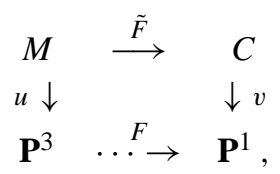

where $C \simeq \mathbf{P}^{1}$ and $\tilde{F}$ is a surjective holomorphic map. Each element of $\Gamma$ induces a bimeromorphic map of $M$ and a biholomorphic map of $C$. Since the group of automorphisms $\gamma$ of $C$ induced by $\Gamma$ which satisfy $v \circ \gamma=v$ is finite, we can choose a normal subgroup $\Gamma_{1}$ of $\Gamma$ with a finite index such that each element $g \in \Gamma_{1}$ induces the identity on $C$. Thus replacing $X=\Omega / \Gamma$ with $X_{1}=\Omega / \Gamma_{1}$, we can assume that the hypersurfaces

$$
S_{t}=\left\{z \in \mathbf{P}^{3}: F(z)=t\right\}, \quad t \in \mathbf{P}^{1},
$$

are irreducible and non-singular outside the base locus $B$ except for a finite number of $S_{t}$ 's, $t=a_{1}, \cdots, a_{s}$. Suppose that $s>0$ and consider a rational function $h(t)$ which has poles exactly on the set $\left\{a_{1}, \cdots, a_{s}\right\}$. Put

$$
S=\bigcup_{j=1}^{s} S_{a_{j}}, \quad D=\bigcup_{j=1}^{s} D_{a_{j}}, \quad D_{t}=\pi\left(S_{t} \cap \Omega\right) .
$$

LEMMA 2.1. $\operatorname{Hex}_{n}(X \backslash D) \geq 4$. 
Proof. Let $\mu: X^{*} \rightarrow X$ be a modification of $X$ with the centers contained in $\pi(\Omega \cap$ $B)$. Let $E^{*}$ be the exceptional divisor on $X^{*}$ and $D^{*}$ be the proper transform of $D$. The map $f$ defines a smooth family

$$
f^{*}: X^{*} \backslash D^{*} \rightarrow \mathbf{P}^{1} \backslash\left\{a_{1}, \cdots, a_{s}\right\}
$$

of compact surfaces. Therefore by Theorem 1.2, we have

$$
\operatorname{Hex}_{n}\left(X^{*} \backslash D^{*}\right) \geq 4 \text {. }
$$

Since $B$ is contained in $D$, the map

$$
\mu: X^{*} \backslash\left(D^{*} \cup E^{*}\right) \rightarrow X \backslash D
$$

is biholomorphic. By Lemma 1.2, we have

$$
4 \leq \operatorname{Hex}_{n}\left(X^{*} \backslash D^{*}\right) \leq \operatorname{Hex}_{n}\left(X^{*} \backslash\left(D^{*} \cup E^{*}\right)\right)=\operatorname{Hex}_{n}(X \backslash D) .
$$

Let $\left[z_{0}: z_{1}: z_{2}: z_{3}\right]$ be a system of homogeneous coordinates on $\mathbf{P}^{3}$. Put

$$
\begin{gathered}
U=\left\{\left[z_{0}: z_{1}: z_{2}: z_{3}\right] \in \mathbf{P}^{3}:\left|z_{0}\right|^{2}+\left|z_{1}\right|^{2}<\left|z_{2}\right|^{2}+\left|z_{3}\right|^{2}\right\}, \\
H_{0}=\left\{\left[z_{0}: z_{1}: z_{2}: z_{3}\right] \in \mathbf{P}^{3}: z_{0}=0\right\}, \quad H_{1}=\left\{\left[z_{0}: z_{1}: z_{2}: z_{3}\right] \in \mathbf{P}^{3}: z_{1}=0\right\}, \\
L_{0}=\left\{\left[z_{0}: z_{1}: z_{2}: z_{3}\right] \in \mathbf{P}^{3}: z_{0}=z_{1}=0\right\} .
\end{gathered}
$$

Let $L$ be a line contained in $\Omega$. Displacing $L$ slightly if necessary, we can assume that there are a neighborhood $U_{L}$ and a biholomorphic map $\tau: U \rightarrow U_{L}$ such that $\pi_{\mid U_{L}}$ is an open embedding and that $\tau\left(L_{0}\right)=L$. Let $\ell=\pi(L)$. We can further assume that $\ell$ intersects each $D_{a_{j}}(j=1, \cdots, s)$ transversely. Note that $\ell$ intersects $D_{t}$ for any $t \in \mathbf{P}^{1}$. Note also that $\tau$ extends to an element of PGL(4) ([K2, Lemma 3.2]). Consider the holomorphic map defined by

$$
\sigma=\pi \circ \tau: U \rightarrow \Omega / \Gamma
$$

Then we see that

$$
U \backslash H_{v} \simeq\left\{\left(x_{1}, x_{2}, x_{3}\right) \in \mathbf{C}^{3}: 1+\left|x_{1}\right|^{2}<\left|x_{2}\right|^{2}+\left|x_{3}\right|^{2}\right\}, \quad v=0,1 .
$$

The $\tau^{-1}\left(S_{a_{j}}\right) \backslash H_{v}$ are biholomorphic to (possibly singular, reducible) affine surfaces in $\mathbf{C}^{3}$. For each $k \in \mathbf{N}$, we consider the following subdomains in $\mathbf{C}^{3}$ :

$$
\begin{aligned}
& G_{1}(k)=\left\{\left(x_{1}, x_{2}, x_{3}\right) \in \mathbf{C}^{3}:\left|x_{1}\right|<k, \quad\left|x_{2}\right|<\sqrt{\frac{k^{2}+3}{2}}, \quad\left|x_{3}\right|<\sqrt{\frac{k^{2}+3}{2}}\right\}, \\
& G_{2}(k)=\left\{\left(x_{1}, x_{2}, x_{3}\right) \in \mathbf{C}^{3}:\left|x_{1}\right|<k, \quad\left|x_{2}\right|<\sqrt{\frac{k^{2}+2}{2}},\left|x_{3}\right|<\sqrt{\frac{k^{2}+2}{2}}\right\},
\end{aligned}
$$




$$
W(k)=G_{1}(k) \backslash\left[G_{2}(k)\right], \quad U(k)=\left\{\left(x_{1}, x_{2}, x_{3}\right) \in \mathbf{C}^{3}:\left|x_{1}\right|<k\right\} .
$$

Since $\tau^{-1}(S)$ coincides with the set of poles of the rational function $(F \circ \tau)^{*} h$, we see by a theorem of Dloussky [D], that the envelope of holomorphy for the étale domain $W(k) \backslash \tau^{-1}(S)$ over $\mathbf{C}^{3}$ is $G_{1}(k) \backslash \tau^{-1}(S)$. Since $\pi_{\mid \Omega \backslash S}: \Omega \backslash S \rightarrow X \backslash D$ is an unramified even covering, we have

$$
\operatorname{Hex}_{3}(\Omega \backslash S) \geq \operatorname{Hex}_{3}(X \backslash D) \geq 4
$$

by Lemmas 1.1 and 2.1 for $n=3$. Hence, by Proposition 1.2, we see that there is a closed subset $A_{v}(k) \subset G_{1}(k) \backslash S$ with $d\left(A_{v}(k)\right) \leq 2$ such that

$$
\tau_{\mid U(k) \backslash H_{v}}: U(k) \backslash H_{v} \rightarrow \Omega \backslash S
$$

extends holomorphically to

$$
\tau_{v}(k): \mathbf{P}^{3} \backslash\left(H_{v} \cup A_{v}(k) \cup T\right) \rightarrow \Omega \backslash S,
$$

where $T$ is a hypersurface in $\mathbf{P}^{3}$ containing $\tau^{-1}(S)$ as an open subset. Letting $k \rightarrow \infty$, we see that $\tau(k)$ extends to

$$
\tau_{v}: \mathbf{P}^{3} \backslash\left(H_{v} \cup A_{v} \cup T\right) \rightarrow \Omega \backslash S,
$$

where $A_{v}=\bigcup_{k} A_{v}(k)$. Thus we see that $\Omega$ contains $\tau_{v}\left(\mathbf{P}^{3} \backslash\left(H_{v} \cup A_{v} \cup T\right)\right)$, and therefore $\Lambda=\mathbf{P}^{3} \backslash \Omega \subset \tau\left(A_{0} \cup A_{1} \cup T\right)$. Here, by the uniqueness of the extension of holomorphic maps, we have $A_{v}(k+1) \cap U(k) \subset A_{v}(k)$. Hence $d\left(A_{v}\right) \leq 2$ holds, and hence we have $d\left(A_{0} \cup A_{1}\right) \leq 2$. Thus Theorem $\mathrm{A}$ is proved.

For the measure of $\Lambda$ outside the algebraic set $S \subset \mathbf{P}^{3}$, we have a little more information.

LEMMA 2.2. For $t \in \mathbf{P}^{1} \backslash\left\{a_{1}, \cdots, s\right\}$, the restricted projection

$$
\pi_{t}=\pi \mid S_{t}: S_{t} \cap \Omega \rightarrow D_{t}
$$

does not extend holomorphically across any point of $S_{t} \cap \Lambda$.

Proof. Take any point $x \in S_{t} \cap \Lambda$. Note that $x$ is a boundary point of $S_{t} \cap \Omega$ in $S_{t}$, since $S_{t} \cap \Lambda$ has no interior point in $S_{t}$ by Theorem A. Suppose that $\pi_{t}$ extends holomorphically to an open connected neighborhood $W$ of $x$ in $S_{t}$. Denote by $\hat{\pi}_{t}$ the extended holomorphic map. Put $y=\hat{\pi}_{t}(x) \in D_{t}$. Since $\pi_{t}: S_{t} \cap \Omega \rightarrow D_{t}$ is a Galois unramified covering, there is a relatively compact subdomain $\Delta$ around $y$ in $D_{t}$ and a relatively compact subdomain $\tilde{\Delta}$ in $S_{t} \cap \Omega$ such that $\pi_{t}^{-1}(\Delta)=\bigcup_{g \in \Gamma} g(\tilde{\Delta})$, where the right-hand side is a disjoint union of relatively compact connected component of $\pi_{t}^{-1}(\Delta)$. Since $\hat{\pi}_{t}$ is continuous, we can assume that $\hat{\pi}_{t}(W) \subset \Delta$. Hence $\pi_{t}(W \cap \Omega)=\hat{\pi}_{t}(W \cap \Omega) \subset \Delta$. Since the Hausdorff dimension of $W \cap \Lambda$ is not more than $2, W \cap \Omega$ is connected. Therefore $W \cap \Omega$ is contained in a connected component of $\pi_{t}^{-1}(\Delta)$. Since each connected component of $\pi_{t}^{-1}(\Delta)$ is relatively compact in $S_{t} \cap \Omega$, we see that the closure $[W \cap \Omega]$ is compact in $S_{t} \cap \Omega$. Hence, for any sequence $\left\{x_{v}\right\}_{v}$, 
$v=1,2, \cdots$, of points in $W \cap \Omega$ which converges to $x \in \Lambda$, we can choose a subsequence of $\left\{x_{v}\right\}_{v}$ which converges to an interior point of $S_{t} \cap \Omega$, which is absurd.

Lemma 2.3. For any $t \in \mathbf{P}^{1} \backslash E$, the Hausdorff dimension $d\left(\left(S_{t} \backslash B\right) \cap \Lambda\right)$ is equal to zero, where $E$ is a subset of $\mathbf{P}^{1}$ with the Lebesgue measure zero, and $B$ is the set of indeterminate of $F: \mathbf{P}^{3} \cdots \rightarrow \mathbf{P}^{1}$.

Proof. In view of the diagram (1), since $u$ is biholomorphic outside $u^{-1}(B)$, it is enough to show that the Hausdorff dimension of $u^{-1}\left(\left(S_{t} \backslash B\right) \cap \Lambda\right)$ is equal to zero for $t \in C \backslash E^{\prime}$, where $E^{\prime}$ is a subset of $C$ with Lebesgue measure zero. Since $B \subset S$, we have by (4) that

$$
d\left(\left(M \backslash u^{-1}(S)\right) \cap u^{-1}(\Lambda)\right)=d\left(\left(\mathbf{P}^{3} \backslash S\right) \cap \Lambda\right)=d(\Lambda \backslash S) \leq 2 .
$$

We consider the continuous map

$$
\tilde{F}^{\prime}: u^{-1}(\Lambda \backslash B) \rightarrow C,
$$

which is the restriction of $\tilde{F}: M \rightarrow C$. Then, by a lemma of Federer (see [S, Lemma 2.7]), we have, for any $k \in \mathbf{N}$, there is a set $E_{k} \subset C$ with Lebesgue measure zero such that

$$
d\left(u^{-1}\left(\left(S_{t} \backslash B\right) \cap \Lambda\right)\right)<\frac{1}{k} \text { for any } t \in C \backslash E_{k} .
$$

Hence, letting $E^{\prime}=\cup_{k=1}^{\infty} E_{k}$, we have the lemma.

Proposition 2.1. There is a set $E \subset \mathbf{P}^{1}$ with Lebesgue measure zero such that, for any $t \in \mathbf{P}^{1} \backslash E,\left(S_{t} \backslash B\right) \cap \Lambda$ is a set of isolated points in $S_{t} \backslash B$.

Proof. Replacing $E$ of Lemma 2.3 by $E \cup\left\{a_{1}, \ldots, a_{s}\right\}$ we take any $t \in \mathbf{P}^{1} \backslash E$. Then $S_{t} \backslash B$ is a non-singular Stein surface. By Lemma 2.3, the envelope of holomorphy for $\left(S_{t} \backslash B\right) \cap \Omega$ over $S_{t} \backslash B$ is $S_{t} \backslash B$. Hence, by Theorem 1.1, $\pi_{t}$ extends to a holomorphic map of $S_{t} \backslash(B \cup A)$ to $D_{t}$, where $A$ is a set of isolated points in $S_{t} \backslash B$. Since $\left(S_{t} \backslash B\right) \cap \Lambda \subset A$ by Lemma 2.2, we see that $\left(S_{t} \backslash B\right) \cap \Lambda$ is a set of isolated points in $S_{t} \backslash B$. Thus we have the proposition.

\section{Proof of Theorem B}

Step 1. Suppose that $\Lambda$ contains uncountably many distinct lines. Since $\Lambda$ is of fibered type, there is a natural injection

$$
\iota: I \hookrightarrow \operatorname{Gr}(4,2) .
$$

We identify the index set $I$ with its image $\iota(I)$. Then there is a projection $\tilde{\iota}: \Lambda \rightarrow I \subset$ $\operatorname{Gr}(4,2)$ defined by $\Lambda \ni z \mapsto \ell \in \operatorname{Gr}(4,2)$, where $z \in \ell$. It is easy to see that $\tilde{\imath}$ is a continuous map and that $I$ is a closed subset in $\operatorname{Gr}(4,2)$, since $\Lambda$ is compact. Put

$$
I^{\prime}=\left\{s \in I: \ell_{s} \subset S\right\}, \quad I^{\prime \prime}=\left\{s \in I: \ell_{s} \not \subset S\right\},
$$


where $S$ is the hypersurface defined by (2). Consider the algebraic subset

$$
\mathcal{B}=\{\ell \in \operatorname{Gr}(4,2): \ell \subset S\} .
$$

By Proposition 2.1, $I^{\prime \prime}$ is a countable set. Let $\mathcal{B}_{0}$ by any irreducible component of $\mathcal{B}$ with $I^{\prime} \cap \mathcal{B}_{0}$ uncountable. Obviously we have $\operatorname{dim} \mathcal{B}_{0} \geq 1$. Let $Z_{0}$ be the tautological fiber space of lines over $\mathcal{B}_{0}$ and $\varepsilon: Z_{0} \rightarrow S$ be the evaluation map. Since $\operatorname{dim} Z_{0}=\operatorname{dim} \mathcal{B}_{0}+1$ and since $2 \leq \operatorname{dim} \varepsilon\left(Z_{0}\right) \leq \operatorname{dim} S=2$, we see that $\varepsilon$ is surjective to an irreducible component $S_{0}$ of $S$. If $\operatorname{dim} \mathcal{B}_{0}=2$, then $\operatorname{dim} \varepsilon^{-1}(x) \geq 1$ for any point $x \in S_{0}$. This implies that on a non-singular model of $S_{0}, \mathcal{B}_{0}$ defines an algebraic family $\mathcal{L}$ whose any two members intersect properly. On the other hand, however, $\mathcal{L}$ contains members, say $C_{1}, C_{2}$, which correspond to lines in $\Lambda^{\prime}$. Since $\Lambda$ is of fibered type, $C_{1}$ and $C_{2}$ do not intersect each other. This is a contradiction. Therefore we have $\operatorname{dim} \mathcal{B}_{0}=1$ and that $\varepsilon\left(Z_{0}\right)$ is a hypersurface in $\mathbf{P}^{3}$.

Step 2. Now we can assume that $\mathcal{B}_{0}$ is $\Gamma$-invariant, by replacing $\Gamma$ with its subgroup of finite index. Let $S_{t}, t \in \mathbf{P}^{1} \backslash E$, be an irreducible hypersurface in $\mathbf{P}^{3}$ of Proposition 2.1 such that $S_{t}$ is non-singular outside the base locus $B$. Put

$$
\Lambda^{\prime}=\bigcup_{s \in I^{\prime}}\left|\ell_{s}\right|, \quad \Lambda^{\prime \prime}=\bigcup_{s \in I^{\prime \prime}}\left|\ell_{s}\right|
$$

Then we have $\Lambda=\Lambda^{\prime} \cup \Lambda^{\prime \prime}$ and $\Lambda^{\prime} \subset S$. Let $v: \tilde{S}_{t} \rightarrow S_{t}$ be the normalization of $S_{t}$. Note that

$$
\left.v\right|_{\tilde{S}_{t} \backslash v^{-1}(B)}: \tilde{S}_{t} \backslash v^{-1}(B) \rightarrow S_{t} \backslash B
$$

is biholomorphic and that $\Gamma$ acts also on $\tilde{S}_{t}$ as an automorphism group. Put $\tilde{v}=\pi \circ v$. Since $S_{t} \cap(B \cup \Lambda)=S_{t} \cap\left(B \cup \Lambda^{\prime \prime}\right)$ and since $d\left(\tilde{S}_{t} \cap v^{-1}\left(B \cup \Lambda^{\prime \prime}\right)\right) \leq 2<5$, the map

$$
\tilde{v}_{t}:=\left.\tilde{v}\right|_{\tilde{S}_{t} \backslash v^{-1}(\Lambda)}: \tilde{S}_{t} \backslash v^{-1}(\Lambda) \rightarrow D_{t}
$$

does not extend holomorphically across any boundary point of $\tilde{S}_{t} \cap v^{-1}(\Lambda)$ by the similar argument to the proof of Lemma 2.2. On the other hand, we note that $\tilde{S}_{t} \backslash v^{-1}(B)$ is a non-singular Stein surface and that the envelope of holomorphy for $\tilde{S}_{t} \backslash v^{-1}(B \cup \Lambda)$ over $\tilde{S}_{t} \backslash v^{-1}(B)$ is $\tilde{S}_{t} \backslash v^{-1}(B)$ itself. By Theorem 1.1, $\tilde{v}_{t}$ extends holomorphically outside of a set $A$ of isolated points in $\tilde{S}_{t} \backslash v^{-1}(B)$, and hence

$$
v^{-1}(\Lambda) \subset A \cup v^{-1}(B) .
$$

Step 3. We claim that, if $B_{1}$ is an irreducible component of $B$ such that $B_{1} \not \subset \Lambda$, then $B_{1} \cap \Lambda$ is a countable set. To verify this claim, we consider the open set $V$ in $v^{-1}\left(B_{1} \backslash \Lambda\right)=$ $v^{-1}\left(B_{1} \cap \Omega\right)$ and a point $\tilde{b} \in \partial V \subset v^{-1}\left(B_{1}\right)$. We assume that $\tilde{b}$ is not only a non-singular point of $\tilde{S}_{t}$ but a non-singular point of $v^{-1}(B)$. Let $W \subset \tilde{S}_{t}$ be a polydisk centered at $\tilde{b}$ with a system of local coordinates $(u, v)$ such that $W=\left\{(u, v) \in \mathbf{C}^{2}:|u|<1,|v|<1\right\}$ and $v^{-1}(B) \cap W=\{v=0\}$. Since $\left(\tilde{S}_{t} \backslash v^{-1}(B)\right) \cap v^{-1}(\Lambda)$ is a set of isolated points in $\tilde{S}_{t} \backslash v^{-1}(B)$, 
there are positive numbers $\varepsilon, \delta$ such that $\{(u, v) \in W:|u|<\delta, \quad|v|=\varepsilon\} \cap \Lambda=\emptyset$ holds, where $\tilde{b}=(0,0)$. Choosing a constant $a$ with $|a|$ small, we can assume that the 1-dimensional $\operatorname{disk}\{(a, v) \in W:|u|<\delta|v| \leq \varepsilon\} \cap \Lambda=\emptyset$. Thus the polydisk $W^{\prime}=\{(u, v) \in W:|u|<$ $\delta,|v|<\varepsilon\}$ is the envelope of holomorphy for a Hartogs domain contained in $\tilde{S}_{t} \backslash v^{-1}(\Lambda)$ over $W$. Then by Theorem 1.1, $W^{\prime} \cap v^{-1}(\Lambda)$ is a set of isolated points. Therefore every boundary point of $V$ is discrete provided that $\tilde{S}_{t}$ and $v^{-1}(B)$ are smooth there. This implies that $B_{1} \cap \Lambda$ is a countable set. Thus our claim is verified. Since any two distinct lines in $\Lambda^{\prime}$ intersect $B$ at distinct points, and since $\Lambda^{\prime}$ contains uncountably many lines, we conclude that there is an irreducible component of $B$ which is contained in $\Lambda$.

Step 4. Put

$$
B^{\prime}=\left\{b \in B: \ell_{b} \subset S \cap \Lambda\right\},
$$

where $\ell_{b}$ is a line in $\Lambda$ passing through the point $b$. Then $B^{\prime}$ is a closed subset of $B$. From step 3 , it follows that $B^{\prime}$ contains an irreducible component of $B$ and that the complement $B \backslash B^{\prime}$ is countable. Therefore, there is a non-degenerate holomorphic map from the irreducible component of $B$ to an irreducible component, say $\mathcal{B}_{0}$, of $\mathcal{B}$. By step 1 and the definition of $B^{\prime}$, we see that the hypersurface $\varepsilon\left(Z_{0}\right)$ is contained in $S \cap \Lambda$. This is absurd however, since $\Omega=\mathbf{P}^{3} \backslash \Lambda \subset \mathbf{P}^{3} \backslash \varepsilon\left(Z_{0}\right)$ and $\Omega$ contains lines. Thus we conclude that $\Lambda$ contains at most countably many distinct lines.

Step 5. Then by a theorem of Hopf on the cardinality of ends, we see that the cardinality of $I$ is at most 2. Hence $X$ is either $\mathbf{P}^{3}$, a Blanchard manifold, or an $L$-Hopf manifold by [K3]. Thus Theorem B is proved.

\section{Holomorphic extension index of a family of surfaces}

In this section, we shall prove Theorem 1.2. Let $X$ be a complex manifold of dimension 3 and $U=\{t \in \mathbf{C}:|t|<1\}$. Suppose that there is a proper holomorphic surjection $f: X \rightarrow U$ with connected fibers and that $f$ is of maximal rank everywhere. Put $X_{t}=f^{-1}(t), t \in U$. Let $b_{j}$ denote the $j$-th Betti number of the manifold $X_{0}$. Put $U_{\varepsilon}=\{t \in \mathbf{C}:|t|<\varepsilon\}$.

4.1. Families of Kähler surfaces. A $C^{\infty}$ differential form $\omega$ on a complex manifold is said to be pluri-closed if $\partial \bar{\partial} \omega=0$ holds. A Hermitian metric on a complex manifold is called a pluri-closed Hermitian metric or pluri-Kähler metric, if the associated Hermitian form is pluriclosed, and the form is called a pluri-closed Hermitian metric form or pluriKähler form.

Then we have the following.

PROPOSITION 4.1. Let $f: X \rightarrow U$ be a complex analytic family of compact Kähler surfaces. Then $f^{-1}\left(U_{\varepsilon}\right)$ admits a pluri-closed Hermitian metric for some $\varepsilon>0$.

PROOF. It is known that a compact complex surface is of Kähler if and only if $b_{1}$ is even. Suppose that $b_{1}$ is even. Let $\Phi: X \rightarrow X_{0} \times \Delta$ be a diffeomorphism, and $\rho: X_{0} \times \Delta \rightarrow$ 
$X_{0}$ be the projection to the first component. Let $\omega_{0}$ be a Kähler metric on $X_{0}$. Let $\tilde{\omega}_{0}$ be the $(1,1)$-component of the $d$-closed form $(\rho \circ \Phi)^{*} \omega_{0}$. Then $\tilde{\omega}_{0}+i d s \wedge d \bar{s}$ gives a pluri-closed Hermitian metric on a neighborhood of $X_{0}$. Thus the proposition follows.

Proposition 4.2. Let $f: X \rightarrow U$ be a smooth family of compact Kähler surfaces. If $X$ is n-probable, then $\operatorname{Hex}_{n}(X) \geq 4$.

Proof. By Propositions 4.1 and 1.3, this follows immediately from Theorem 1.1.

4.2. Families of surfaces with odd $b_{1}>1$. First consider the case where $X_{0}$ is minimal, i.e, free from the exceptional curves of the first kind. In this case, there is $\varepsilon>0$ such that the $X_{t}$ 's are minimal for all $t \in U_{\varepsilon}$. Thus we can assume that every member $X_{t}$ is minimal from the beginning. Main part of the argument in the following is the relative variant of [K1, Section 7(B)].

Proposition 4.3. We can choose $\varepsilon>0$ and $N \in \mathbf{N}$ so that there is a proper finite holomorphic map

$$
\Phi: f^{-1}\left(U_{\varepsilon}\right) \rightarrow\left(\left(\mathbf{C}^{N} \backslash\{O\}\right) \times U_{\varepsilon}\right) /\langle\alpha\rangle
$$

where $\alpha$ is a holomorphic automorphism of $\left(\mathbf{C}^{N} \backslash\{O\}\right) \times U_{\varepsilon}$ of the form

$$
\alpha:\left(z_{1}, \cdots z_{N}, t\right) \mapsto\left(\alpha(t) z_{1}, \cdots, \alpha(t) z_{N}, t\right)
$$

satisfying $|\alpha(t)|<1$ for $t \in U_{\varepsilon}$.

Proof. The direct image sheaf $R^{0} f_{*} \Omega_{X / \Delta}^{1}$ is free on $\Delta$, since the rank of the coherent sheaf is constant given by $b_{1}=2 \operatorname{dim} H^{0}\left(X_{t}, \Omega_{X_{t}}^{1}\right)+1$. Put $g=\operatorname{rank} R^{0} f_{*} \Omega_{X / \Delta}^{1}$. Since every holomorphic 1-form on compact surfaces are $d$-closed, we have the relative Albanese map

$$
\Psi: X \rightarrow\left(\mathbf{C}^{g} \times \Delta\right) / \mathcal{G}, \quad \mathcal{G} \simeq \mathbf{Z}^{2 g}
$$

By [U, Proposition 9.19], Albanese maps of surfaces with odd $b_{1}>1$ have non-singular images, connected fibers, and multiple elliptic curves as singular fibers. Therefore $\Psi$ also has the same properties. Put $M=\Psi(X)$ and $C_{t}=\Psi\left(X_{t}\right)$. Letting $\mu: M \rightarrow \Delta$ be the projection to the 2 nd component, we have a commutative diagram

$$
\begin{aligned}
X \stackrel{\Psi}{\rightarrow} & M \\
f \searrow & \downarrow \mu \\
& \Delta,
\end{aligned}
$$

where $f=\mu \circ \Psi$ and $C_{t}=\mu^{-1}(t), t \in \Delta$. The genus of $C_{t}$ is equal to $g$. Let $\sigma: U_{\varepsilon} \rightarrow M$ be a section of $\mu \mid M_{\varepsilon}$ for some $\varepsilon>0$, where $M_{\varepsilon}=\mu^{-1}\left(U_{\varepsilon}\right)$. Put $X_{\varepsilon}=f^{-1}\left(U_{\varepsilon}\right)$ and consider 
the following commutative diagram,

$$
\begin{aligned}
& \rightarrow \quad H^{1}\left(X_{\varepsilon}, \mathcal{O}_{X_{\varepsilon}}\right) \quad \stackrel{e}{\rightarrow} \quad H^{1}\left(X_{\varepsilon}, \mathcal{O}_{X_{\varepsilon}}^{*}\right) \quad \stackrel{c_{1}}{\rightarrow} H^{2}\left(X_{\varepsilon}, \mathbf{Z}\right) \quad \rightarrow \\
& j \uparrow \quad \uparrow \quad \| \\
& \rightarrow H^{1}\left(X_{\varepsilon}, f^{-1}\left(\mathcal{O}_{U_{\varepsilon}}\right)\right) \stackrel{i}{\rightarrow} H^{1}\left(X_{\varepsilon}, f^{-1}\left(\mathcal{O}_{U_{\varepsilon}}^{*}\right)\right) \rightarrow H^{2}\left(X_{\varepsilon}, \mathbf{Z}\right) \rightarrow .
\end{aligned}
$$

Here $f^{-1}\left(\mathcal{O}_{U_{\varepsilon}}\right)$ and $f^{-1}\left(\mathcal{O}_{U_{\varepsilon}}^{*}\right)$ are the set theoretic inverse of germs. The homomorphism $j$ is the map induced by the natural inclusion. Since $R^{2} f_{*} \mathcal{O}_{X}$ is free with rank $=p_{g}$, we have $\left(R^{1} f_{*} \mathcal{O}_{X}\right)_{t} \simeq H^{1}\left(X_{t}, \mathcal{O}_{X_{t}}\right) \simeq \mathbf{C}^{b_{1}}$ for any $t \in \Delta$. Since we know that

$$
0 \rightarrow H^{0}\left(S, d \mathcal{O}_{S}\right) \rightarrow H^{1}(S, \mathbf{C}) \rightarrow H^{1}\left(S, \mathcal{O}_{S}\right) \rightarrow 0
$$

is exact on any compact surface $S$, we see that

$$
R^{1} f_{*}\left(f^{-1}\left(\mathcal{O}_{U_{\varepsilon}}\right)\right) \simeq R^{1} f_{*} \mathbf{C} \otimes_{\mathbf{C}} \mathcal{O}_{U_{\varepsilon}} \rightarrow R^{1} f_{*} \mathcal{O}_{X_{\varepsilon}}
$$

is surjective. Therefore

$$
0 \rightarrow H^{0}\left(X_{\varepsilon}, \mathcal{O}_{X_{\varepsilon}} / f^{-1}\left(\mathcal{O}_{U_{\varepsilon}}\right)\right) \rightarrow H^{1}\left(X_{\varepsilon}, f^{-1}\left(\mathcal{O}_{U_{\varepsilon}}\right)\right) \rightarrow H^{1}\left(X_{\varepsilon}, \mathcal{O}_{X_{\varepsilon}}\right) \rightarrow 0
$$

is exact.

LEMMA 4.1. The pull-back $\Psi^{*} F \in H^{1}\left(X_{\varepsilon}, \mathcal{O}_{X_{\varepsilon}}^{*}\right)$ of any line bundle $F \in$ $H^{1}\left(M_{\varepsilon}, \mathcal{O}_{M_{\varepsilon}}^{*}\right)$ has a representative in $H^{1}\left(X_{\varepsilon}, f^{-1}\left(\mathcal{O}_{U_{\varepsilon}}^{*}\right)\right)$.

PROOF. Since

$$
f^{*}: H^{2}\left(M_{\varepsilon}, \mathbf{C}\right) \rightarrow H^{2}\left(X_{\varepsilon}, \mathbf{C}\right)
$$

is a zero-map, we see that $c_{1}\left(\Psi^{*} F^{\otimes m}\right)=0$ for some $m \in \mathbf{N}$. Then, by (5) and (6), there is an element $\eta \in H^{1}\left(X_{\varepsilon}, f^{-1}\left(\mathcal{O}_{U_{\varepsilon}}\right)\right)$ such that $e \circ j(\eta)=\Psi^{*} F$. Hence $i(\eta)$ is the desired element.

Take a section $\sigma: U_{\varepsilon} \rightarrow M_{\varepsilon}$ and regard the image $D=\sigma\left(U_{\varepsilon}\right)$ as an effective divisor on $M_{\varepsilon}$. By Lemma 4.1, the pull-back $\Psi^{*}\left([D]^{\otimes m}\right)$ of the $m$-th multiple of the line bundle $[D]$ is flat on each $X_{t}$. Let

$$
\rho: H_{1}\left(X_{\varepsilon}, \mathbf{Z}\right) \rightarrow \Gamma\left(X_{\varepsilon}, f^{-1}\left(\mathcal{O}_{U_{\varepsilon}}^{*}\right)\right)=\Gamma\left(U_{\varepsilon}, \mathcal{O}_{U_{\varepsilon}}^{*}\right)
$$

be the associated group representation. Choose $m \in \mathbf{N}$ such that $\rho\left(H_{1}\left(X_{\varepsilon}, \mathbf{Z}\right)_{\text {tor }}\right)=\{1\}$. Let $\left\{\gamma_{0}, \gamma_{1}, \cdots, \gamma_{2 g}\right\}$ be a Betti basis of $H_{1}\left(X_{\varepsilon}, \mathbf{Z}\right)$ such that the $f_{*}\left(\gamma_{j}\right)$ form a Betti basis of $H_{1}\left(M_{\varepsilon}, \mathbf{Z}\right)$ and $f_{*}\left(\gamma_{0}\right)=0$. We consider the line bundle $\xi \in H^{1}\left(M_{\varepsilon}, \mu^{-1}\left(\mathcal{O}_{U_{\varepsilon}}^{*}\right)\right)$ associated with the representation $\tau$ defined by

$$
\tau\left(f_{*} \gamma_{j}\right)=\rho\left(\gamma_{j}\right), \quad j=1, \cdots, 2 g .
$$

Then $F=\Psi^{*}\left([D]^{\otimes m} \otimes \xi^{-1}\right) \in H^{1}\left(X_{\varepsilon}, f^{-1}\left(\mathcal{O}_{U_{\varepsilon}}^{*}\right)\right)$ is the line bundle of which the associated representation $\rho_{1}: H_{1}\left(X_{\varepsilon}, \mathbf{Z}\right) \rightarrow \Gamma\left(U_{\varepsilon}, \mathcal{O}_{U_{\varepsilon}}^{*}\right)$ is given by

$$
\rho_{1}\left(\gamma_{0}\right)=\rho\left(\gamma_{0}\right), \quad \rho_{1}\left(\gamma_{j}\right)=1, \quad j=1, \cdots, 2 g .
$$


Put $\alpha=\rho_{1}\left(\gamma_{0}\right) \in \Gamma\left(U_{\varepsilon}, \mathcal{O}_{U_{\varepsilon}}^{*}\right)$. Then the image of the group representation $\rho_{1}$ is an infinite cyclic group in $\Gamma\left(U_{\varepsilon}, \mathcal{O}_{U_{\varepsilon}}^{*}\right)$ generated by $\alpha$. Note that $\alpha=\alpha(t)$ is a holomorphic function on $U_{\varepsilon}$ and we have $|\alpha(t)| \neq 1$ for any $t \in U_{\varepsilon}$. Here we can assume that $|\alpha(t)|<1$ for any $t \in \Delta$, replacing $\alpha$ with $\alpha^{-1}$ if necessary. Fix $m$ to be a sufficiently large number. Then, since

$$
\operatorname{dim} \Gamma\left(X_{t}, F_{\mid X_{t}}\right)=\operatorname{dim} \Gamma\left(C_{t},\left([D]^{\otimes m} \otimes \xi^{-1}\right)_{\mid C_{t}}\right)=2-2 g+m=3-b_{1}+m
$$

is independent of $t, R^{0} f_{*} F$ is free on $U_{\varepsilon}$. Put $N=3-b_{1}+m$. Let $\mathcal{G}$ be the cyclic group generated by a holomorphic automorphism of $\left(\mathbf{C}^{N} \backslash\{O\}\right) \times U_{\varepsilon}$ of the form

$$
\alpha:\left(z_{1}, \cdots z_{N}, t\right) \mapsto\left(\alpha(t) z_{1}, \cdots, \alpha(t) z_{N}, t\right) .
$$

Let $\left\{\varphi_{1}, \cdots, \varphi_{N}\right\}$ be the set of generators of $\left(R^{0} f_{*} F\right)_{0}$. Then the map

$$
\Phi: x \mapsto\left(\varphi_{1}(x), \cdots, \varphi_{N}(x)\right), \quad x \in X_{\varepsilon}
$$

defines the desired holomorphic map for a sufficiently small $\varepsilon>0$.

REMARK 4.1. By the construction above, we have the following commutative diagram,

$$
\begin{array}{ccc}
X_{\varepsilon} & \stackrel{\Phi}{\longrightarrow} & \left(\left(\mathbf{C}^{N} \backslash\{O\}\right) \times U_{\varepsilon}\right) /\langle\alpha\rangle \\
f \downarrow & & \downarrow \varpi \\
U_{\varepsilon} & \stackrel{p}{\longleftarrow} & \mathbf{P}^{N-1} \times U_{\varepsilon},
\end{array}
$$

where $\varpi$ is the natural projection, and $p$ is the projection to the 2 nd component. If $m \geq$ $2 g+1=b_{1}, \Phi$ induces a holomorphic embedding $M_{\varepsilon} \hookrightarrow \mathbf{P}^{N-1} \times U_{\varepsilon}$.

Proposition 4.4. There is a Stein space $Z$ of dimension 3 and an analytic subset $C \subset Z$ of dimension 1 such that $Z \backslash C$ is non-singular, and is an infinite cyclic unramified covering of $X_{\varepsilon}$. In particular, $X_{\varepsilon}$ is $n$-probable.

PROOF. Let $\tilde{X}_{\varepsilon}$ the infinite unramified covering corresponding to the kernel of

$$
\Psi_{*}: \pi_{1}\left(X_{\varepsilon}\right) \rightarrow \pi_{1}\left(\left(\left(\mathbf{C}^{N} \backslash\{O\}\right) \times U_{\varepsilon}\right) /\langle\alpha\rangle\right) .
$$

Put $W_{\varepsilon}=q^{-1}\left(\Psi\left(X_{\varepsilon}\right)\right)$, where $q:\left(\mathbf{C}^{N} \backslash\{O\}\right) \times U_{\varepsilon} \rightarrow\left(\left(\mathbf{C}^{N} \backslash\{O\}\right) \times U_{\varepsilon}\right) /\langle\alpha\rangle$ is the canonical projection. Then $\Psi$ lifts to

$$
\tilde{\Psi}: \tilde{X}_{\varepsilon} \rightarrow W_{\varepsilon}
$$

a finite branched covering. Note that we can extend $W_{\varepsilon}$ by attaching 1-dimensional subset $C=\{O\} \times U_{\varepsilon}$ to a closed analytic subvariety $\hat{W}_{\varepsilon}$ in $\mathbf{C}^{N} \times U_{\varepsilon}$. Therefore, by the continuation theorem of Grauert-Remmert, $\tilde{X}_{\varepsilon}$ also can be extended to a complex analytic space $Z_{\varepsilon}$ which is proper over $\hat{W}_{\varepsilon}$. Hence $Z_{\varepsilon}$ is a Stein space. Since the deck transformation of $\tilde{X}_{\varepsilon}$ which corresponds to $\alpha$ acts on $Z_{\varepsilon}$ as a contracting automorphism on each fiber of $\varpi$ and fixes each point on $C$, we see that $Z_{\varepsilon} \backslash \tilde{X}_{\varepsilon}$ is a 1-dimensional analytic set biholomorphic to $C$. Thus the proposition is proved. 
PROPOSITION 4.5. Let $f: X \rightarrow U$ be a smooth family of compact non-Kähler surfaces with odd $b_{1}>1$. Suppose that $X$ is n-probable. If every member of the family is minimal, then $\operatorname{Hex}_{n}(X)=\infty$, otherwise $\operatorname{Hex}_{n}(X)=4$.

Proof. Suppose that every member is minimal. Then by Propositions 4.4 and 4.3 , this follows from Proposition 1.3, since the image manifold $\Phi\left(f^{-1}\left(U_{\varepsilon}\right)\right)$ is a family of elliptic bundle over a curve of positive genus, and hence

$$
\operatorname{Hex}_{n}\left(f^{-1}\left(U_{\varepsilon}\right)\right)=+\infty .
$$

Since the exceptional curves of the first kind constitute a complex analytic family of submanifolds over $U_{\varepsilon}$, we can blow down such curves simultaneously to obtain another smooth family of compact surfaces with the same $b_{1}$. By a finite steps of blowing-downs, we obtain a family of minimal surfaces. Thus in this case we have $\operatorname{Hex}_{n}(X)=4$.

4.3. Families of surfaces with $b_{1}=1$. The aim of this subsection is to prove that Proposition 4.5 holds true also in the case $b_{1}=1$, by showing the existence of a pluri-closed Hermitian metric. It is known by Gauduchon [G] that every compact complex surface admits a pluri-closed Hermitian metric form. Therefore what have to do is to check the existence of such forms on a neighborhood of each member of the smooth family.

We shall begin the argument in a little more general setting.

PROPOSITION 4.6. On a complex manifold $Y$, we have the following exact sequence of sheaves:

$$
0 \longrightarrow \mathcal{O}_{Y} / i \mathbf{R} \stackrel{\alpha_{1}}{\longrightarrow} \mathcal{R}_{Y} \oplus \Omega_{Y}^{1} \stackrel{\alpha_{2}}{\longrightarrow} \mathcal{A}_{Y}^{1,0} \stackrel{\alpha_{3}}{\longrightarrow} \mathcal{H}_{Y} \longrightarrow 0
$$

where

$$
\begin{aligned}
i \mathbf{R} & =\text { the constant sheaf of the pure imaginary number on } Y, \\
\mathcal{O}_{Y} & =\text { the sheaf of germs of holomorphic functions on } Y, \\
\mathcal{R}_{Y} & =\text { the sheaf of germs of real-valued } \mathbf{C}^{\infty} \text { functions on } Y, \\
\Omega_{Y}^{1} & =\text { the sheaf of germs of holomorphic } 1 \text {-forms on } Y, \\
\mathcal{A}_{Y}^{p, q} & =\text { the sheaf of germs of } C^{\infty}(p, q) \text {-forms on } Y, \\
\mathcal{H}_{Y} & =\operatorname{Im}\left(\mathcal{A}_{Y}^{1,0} \stackrel{\alpha_{3}}{\longrightarrow} \mathcal{A}_{Y}^{1,1}\right)
\end{aligned}
$$

and the sheaf homomorphisms are defined by

$$
\alpha_{1}(\varphi)=(\varphi+\bar{\varphi},-d \varphi), \quad \alpha_{2}(\varphi, \theta)=\partial \varphi+\theta, \quad \alpha_{3}(\eta)=\partial \bar{\eta}+\bar{\partial} \eta .
$$

Proof. (1) It is clear that $\alpha_{1}$ is injective. (2) Proof of Im $\alpha_{1} \subset \operatorname{Ker} \alpha_{2}$. Take $\varphi \in \mathcal{O}_{Y}$. Then $\alpha_{2} \circ \alpha_{1}(\varphi)=\alpha_{2}(\varphi+\bar{\varphi},-d \varphi)=\partial(\varphi+\bar{\varphi})-d \varphi=\partial \varphi-\partial \varphi=0$. (3) Proof of $\operatorname{Ker} \alpha_{2} \subset \operatorname{Im} \alpha_{1}$. Take any germ $(\varphi, \theta) \in \operatorname{Ker} \alpha_{2}$. Since $\partial \varphi+\theta=0$, there is an element $h \in \mathcal{O}_{Y}$ such that $\theta=-d h$. Therefore $\partial(\varphi-h)=0$. Hence there is an element $g \in \mathcal{O}_{Y}$ such that $\varphi=h+\bar{g}$. Since $\varphi$ is real, $g-h$ is a certain real constant function. Therefore we can 
replace $h$ with a suitable element of $\mathcal{O}_{Y}$ so that the equality $\varphi=h+\bar{h}$ holds. The $h$ defines a desired class $[h] \in \mathcal{O}_{Y} / i \mathbf{R}$. (4) The implication Im $\alpha_{2} \subset \operatorname{Ker} \alpha_{3}$ is clear. (5) Proof of $\operatorname{Ker} \alpha_{3} \subset \operatorname{Im} \alpha_{2}$. Fix any point $y \in Y$ and any germ $\eta \in \operatorname{Ker} \alpha_{3}$ in the stalk over $y$. We put $\omega=i \partial \bar{\eta}$. Since $\omega=\bar{\omega}$ and $\partial \omega=0$, we see that $\omega$ is a $C^{\infty}(1,1)$-form with $d \omega=0$. Hence there is a $C^{\infty}$ real-valued function $u$ such that $\omega=i \partial \bar{\partial} u$. Put $\theta=\eta-\partial u$. Then the germ $(u, \theta) \in \mathcal{R}_{Y} \oplus \Omega_{Y}^{1}$ has the image $\eta$ by the sheaf homomorphism $\alpha_{2}$. (6) Surjectivity of $\alpha_{3}$ is obvious by definition.

Let $X$ be a complex manifold of dimension $n+1$ and $\Delta=\{t \in \mathbf{C}:|t|<1\}$. Let $f: X \rightarrow \Delta$ be a proper surjective holomorphic map which is of maximal rank everywhere on $X$. Then $X_{t}=f^{-1}(t)(t \in \Delta)$ is a non-singular compact complex manifolds of dimension $n$. Assume that the central fiber $X_{0}=f^{-1}(0)$ satisfies the following three conditions:

$$
H^{2}\left(X_{0}, \mathcal{O}_{X_{0}}\right)=0,
$$$$
H^{3}\left(X_{0}, \mathcal{O}_{X_{0}}\right)=0 \text {, }
$$

$$
H^{2}\left(X_{0}, \Omega_{X_{0}}^{1}\right)=0 \text {. }
$$

Let $\iota: X_{0} \rightarrow X$ be the inclusion map. By Proposition 4.6, we have the following commutative diagram of sheaves on $X$ :

$$
\begin{array}{ccccccccc}
0 & \rightarrow & \mathcal{O}_{X} / i \mathbf{R} & \rightarrow & \mathcal{R}_{X} \oplus \Omega_{X}^{1} & \rightarrow & \mathcal{G}_{X} & \rightarrow & 0 \\
& & \downarrow & & & \downarrow & & \downarrow & \\
0 & \rightarrow & \mathcal{O}_{X_{0}} / i \mathbf{R} & \rightarrow & \mathcal{R}_{X_{0}} \oplus \Omega_{X_{0}}^{1} & \rightarrow & \mathcal{G}_{X_{0}} & \rightarrow & 0
\end{array}
$$

and

$$
\begin{array}{ccccccccc}
0 & \rightarrow & \mathcal{G}_{X} & \rightarrow & \mathcal{A}_{X}^{1,0} & \rightarrow & \mathcal{H}_{X} & \rightarrow & 0 \\
& & \downarrow & & \downarrow & & \downarrow & & \\
0 & \rightarrow & \mathcal{G}_{X_{0}} & \rightarrow & \mathcal{A}_{X_{0}}^{1,0} & \rightarrow & \mathcal{H}_{X_{0}} & \rightarrow & 0,
\end{array}
$$

where

$$
\mathcal{G}_{X}=\operatorname{coker}\left\{\mathcal{O}_{X} / i \mathbf{R} \stackrel{\alpha_{1}}{\rightarrow} \mathcal{R}_{X} \oplus \Omega_{X}^{1}\right\}, \quad \mathcal{G}_{X_{0}}=\operatorname{coker}\left\{\mathcal{O}_{X_{0}} / i \mathbf{R} \stackrel{\alpha_{1}}{\rightarrow} \mathcal{R}_{X_{0}} \oplus \Omega_{X_{0}}^{1}\right\}
$$

LEMMA 4.2. The natural map $R^{q} f_{*} \Omega_{X}^{1} \rightarrow R^{q} f_{*} \Omega_{X_{0}}^{1}$ is surjective for $q=1$ and injective for $q=2$.

Proof. Consider the exact sequences of sheaves

$$
\begin{gathered}
0 \rightarrow f^{*} \Omega_{\Delta}^{1} \rightarrow \Omega_{X}^{1} \rightarrow \Omega_{X / \Delta}^{1} \rightarrow 0, \\
0 \rightarrow f^{*}(m) \Omega_{X / \Delta}^{1} \rightarrow \Omega_{X / \Delta}^{1} \rightarrow \Omega_{X_{0}}^{1} \rightarrow 0,
\end{gathered}
$$


where $m$ is the maximal ideal of the stalk $\mathcal{O}_{\Delta 0}$ at $0 \in \Delta$. The natural map $R^{q} f_{*} \Omega_{X}^{1} \rightarrow$ $R^{q} f_{*} \Omega_{X_{0}}^{1}$ is the composition of the natural maps

$$
R^{q} f_{*} \Omega_{X}^{1} \stackrel{u_{q}}{\rightarrow} R^{q} f_{*} \Omega_{X / \Delta}^{1} \stackrel{v_{q}}{\rightarrow} R^{q} f_{*} \Omega_{X_{0}}^{1} .
$$

Since

$$
R^{2} f_{*} f^{*}\left(\Omega_{\Delta}^{1}\right)=R^{2} f_{*} \mathcal{O}_{X} \otimes \mathcal{O}_{\Delta} \Omega_{\Delta}^{1}=0
$$

by the assumption (8), $u_{1}$ is surjective and $u_{2}$ is injective by (13). Since

$$
R^{2} f_{*} \Omega_{X_{0}}^{1}=H^{2}\left(X_{0}, \Omega_{X_{0}}^{1}\right)=0
$$

by the assumption (10), we have

$$
R^{2} f_{*} \Omega_{X / \Delta}^{1}=0
$$

by (14) and Nakayama's Lemma. Hence $v_{1}$ is surjective and $v_{2}$ is injective by (14). Thus we have the lemma.

LEMma 4.3. $\iota^{*}: R^{2} f_{*} \mathcal{O}_{X} / i \mathbf{R} \rightarrow R^{2} f_{*} \mathcal{O}_{X_{0}} / i \mathbf{R}$ is an isomorphism.

Proof. By the assumption (9) and by the exact sequences

$$
0 \rightarrow i \mathbf{R} \rightarrow \mathcal{O}_{X} \rightarrow \mathcal{O}_{X} / i \mathbf{R} \rightarrow 0, \quad 0 \rightarrow i \mathbf{R} \rightarrow \mathcal{O}_{X_{0}} \rightarrow \mathcal{O}_{X_{0}} / i \mathbf{R} \rightarrow 0,
$$

we have the isomorphisms

$$
R^{2} f_{*}\left(\mathcal{O}_{X} / i \mathbf{R}\right) \simeq R^{3} f_{*} i \mathbf{R} \simeq R^{2} f_{*}\left(\mathcal{O}_{X_{0}} / i \mathbf{R}\right) .
$$

LEMMA 4.4. $\quad R^{1} f_{*} \mathcal{G}_{X} \rightarrow R^{1} f_{*} \mathcal{G}_{X_{0}}$ is surjective.

Proof. Since

$$
R^{1} f_{*} \mathcal{R}_{X}=R^{1} f_{*} \mathcal{R}_{X_{0}}=0
$$

we have by (11) the diagram

$$
\begin{aligned}
& \rightarrow R^{1} f_{*} \Omega_{X}^{1} \rightarrow R^{1} f_{*} \mathcal{G}_{X} \rightarrow R^{2} f_{*}\left(\mathcal{O}_{X} / i \mathbf{R}\right) \rightarrow R^{2} f_{*} \Omega_{X}^{1} \quad \rightarrow \\
& i_{0} \downarrow \quad i_{1} \downarrow \quad i_{2} \downarrow \\
& \rightarrow R^{1} f_{*} \Omega_{X_{0}}^{1} \rightarrow R^{1} f_{*} \mathcal{G}_{X_{0}} \rightarrow R^{2} f_{*}\left(\mathcal{O}_{X_{0}} / i \mathbf{R}\right) \rightarrow R^{2} f_{*} \Omega_{X_{0}}^{1} \rightarrow,
\end{aligned}
$$

where the low sequences are exact. Then by Lemmas 4.2, 4.3, we have the lemma.

LEMMA 4.5. $\quad R^{0} f_{*} \mathcal{H}_{X} \rightarrow R^{0} f_{*} \mathcal{H}_{X_{0}}$ is surjective.

Proof. By (12), we have the diagram,

$$
\begin{aligned}
& \rightarrow \quad R^{0} f_{*} \mathcal{A}_{X}^{1,0} \rightarrow R^{0} f_{*} \mathcal{H}_{X} \quad \rightarrow \quad R^{1} f_{*} \mathcal{G}_{X} \quad \rightarrow \quad R^{1} f_{*} \mathcal{A}_{X}^{1,0} \rightarrow
\end{aligned}
$$

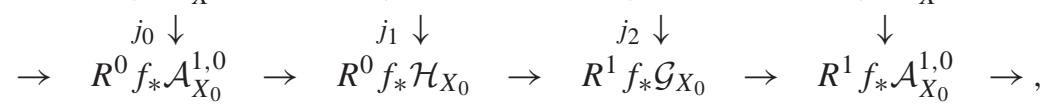


where the low sequences are exact. Since

$$
R^{1} f_{*} \mathcal{A}_{X}^{1,0}=R^{1} f_{*} \mathcal{A}_{X_{0}}^{1,0}=R^{1} f_{*}\left(\mathcal{A}_{X}^{1,0}\left(-X_{0}\right)\right)=0
$$

we have the lemma by Lemma 4.4.

PROPOSITION 4.7. Let $f: X \rightarrow U$ be a smooth family of compact complex manifolds of dimension $n$. Suppose that the central fiber $X_{0}=f^{-1}(0)$ admits a pluri-closed Hermitian metric form and satisfies

$$
H^{2}\left(X_{0}, \mathcal{O}_{X_{0}}\right)=H^{3}\left(X_{0}, \mathcal{O}_{X_{0}}\right)=H^{2}\left(X_{0}, \Omega_{X_{0}}^{1}\right)=0
$$

Then $f^{-1}\left(U_{\varepsilon}\right)$ admits a pluri-closed Hermitian metric form for some $\varepsilon>0$.

Proof. The pluri-closed Hermitian metric form on $X_{0}$ defines an element $\omega \in$ $R^{0} f_{*} \mathcal{H}_{X_{0}}$. Then by Lemma 4.5 , there is a pluri-closed Hermitian form $\tilde{\omega}$ on $f^{-1}\left(U_{\varepsilon}\right)$ for some $\varepsilon>0$ such that $\tilde{\omega}_{\mid X_{0}}=\omega$. Then $\tilde{\omega}+i d t \wedge d \bar{t}$ would be a pluri-closed Hermitian metric form on $f^{-1}\left(U_{\varepsilon_{1}}\right)$ for some $0<\varepsilon_{1}<\varepsilon$.

Now we go back to the original situation. Let $X$ be a complex manifold of dimension 3 and $\Delta=\{t \in \mathbf{C}:|t|<1\}$. Let $f: X \rightarrow \Delta$ be a proper surjective holomorphic map which is of maximal rank everywhere on $X$. Then each $X_{t}=f^{-1}(t)(t \in \Delta)$ is a non-singular compact complex surface.

PROPOSITION 4.8. Let $f: X \rightarrow U$ be a smooth family of compact complex surfaces with $b_{1}=1$. Then $f^{-1}\left(U_{\varepsilon}\right)$ admits a pluri-closed Hermitian metric form for some $\varepsilon>0$.

Proof. Since $\operatorname{dim} X_{t}=2$, we have always $H^{3}\left(X_{t}, \mathcal{O}_{X_{t}}\right)=0$. Since $b_{1}=1$, we have also $H^{2}\left(X_{0}, \mathcal{O}_{X_{0}}\right)=0$ and $H^{2}\left(X_{0}, \Omega_{X_{0}}^{1}\right)=H^{0}\left(X_{0}, \Omega_{X_{0}}^{1}\right)=0$. Further by a theorem of Gauduchon [G], there is an element $\omega \in R^{0} f_{*} \mathcal{H}_{X_{0}}$ which defines a pluri-closed Hermitian metric form on $X_{0}$. Hence the proposition follows from Proposition 4.7.

PROPOSITION 4.9. Let $f: X \rightarrow U$ be a smooth family of compact complex surfaces with $b_{1}=1$. If $X$ is $n$-probable, then $\operatorname{Hex}_{n}(X) \geq 4$ holds.

Proof. By Propositions 4.8 and 1.3, this follows immediately from Theorem 1.1.

Proof of Theorem 1.2. Combine Propositions 4.2, 4.5 and 4.9.

\section{On the structure of $\Lambda$}

If $X=\Omega / \Gamma$ is a flat twistor space over a conformally flat differentiable 4-manifold, it is clear that $\mathbf{P}^{3} \backslash \Omega$ is of fibered type. The complex analytic connected sum of flat twistor spaces is no longer a flat twistor space. We can show, however, the following.

Proposition 5.1. Suppose that $X_{1}=\Omega_{1} / \Gamma_{1}$ and $X_{2}=\Omega_{2} / \Gamma_{2}$ are compact quotients of large domains in $\mathbf{P}^{3}$. If both $\Lambda_{1}$ and $\Lambda_{2}$ are of fibered type, then the complex analytic 
connected sum $X_{1} \# X_{2}$ is also a compact quotient of a large domain $\Omega$ in $\mathbf{P}^{3}$ such that the complement $\mathbf{P}^{3} \backslash \Omega$ is of fibered type.

We recall the argument of Maskit [M] (see also [K5]). Fix a system of homogeneous coordinates $z=\left[z_{0}: z_{1}: z_{2}: z_{3}\right]$ on $\mathbf{P}^{3}$ and we consider the real hypersurface

$$
\Sigma=\left\{z \in \mathbf{P}^{3}:\left|z_{0}\right|^{2}+\left|z_{1}\right|^{2}=\left|z_{2}\right|^{2}+\left|z_{3}\right|^{2}\right\} .
$$

Fix $0<\varepsilon<1$ and define

$$
\begin{aligned}
W & =\left\{z \in \mathbf{P}^{3}: \varepsilon\left(\left|z_{0}\right|^{2}+\left|z_{1}\right|^{2}\right)<\left|z_{2}\right|^{2}+\left|z_{3}\right|^{2}<\varepsilon^{-1}\left(\left|z_{0}\right|^{2}+\left|z_{1}\right|^{2}\right)\right\}, \\
W_{1} & =\left\{z \in \mathbf{P}^{3}:\left|z_{0}\right|^{2}+\left|z_{1}\right|^{2}<\varepsilon^{-1}\left(\left|z_{2}\right|^{2}+\left|z_{3}\right|^{2}\right)\right\}, \\
W_{1}^{\prime} & =\left\{z \in \mathbf{P}^{3}:\left|z_{0}\right|^{2}+\left|z_{1}\right|^{2}<\left|z_{2}\right|^{2}+\left|z_{3}\right|^{2}\right\}, \\
W_{2} & =\left\{z \in \mathbf{P}^{3}:\left|z_{2}\right|^{2}+\left|z_{3}\right|^{2}<\varepsilon^{-1}\left(\left|z_{0}\right|^{2}+\left|z_{1}\right|^{2}\right),\right. \\
W_{2}^{\prime} & \left.=\left\{z \in \mathbf{P}^{3}:\left|z_{2}\right|^{2}+\left|z_{3}\right|^{2}<\left|z_{0}\right|^{2}+\left|z_{1}\right|^{2}\right\} .\right\} .
\end{aligned}
$$

We prepare also a copy $U$ of $W_{1}$. Suppose that there are open holomorphic embeddings $j_{v}: W_{v} \rightarrow X_{v}$. Then the complex analytic connected sum $X_{1} \# X_{2}=\operatorname{Sum}\left(X_{1}, X_{2}, j_{1}, j_{2}, \Sigma\right)$ of $X_{1}$ and $X_{2}$ is the union $X_{1}^{\sharp} \cup X_{2}^{\sharp}, X_{v}^{\sharp}=X_{v} \backslash j_{v}\left(W_{v} \backslash W\right)$, where $j_{1}(x) \in j_{1}(W), x \in W$, is identified with $j_{2}(x) \in j_{2}(W)$. Let $\check{j}_{v}: W_{v} \rightarrow \Omega_{v} \subset \mathbf{P}^{3}$ be a lift of $j_{v}$. Then $\breve{j}_{v}$ extends to an element of PGL(4) by [K2, Lemma 3.2]. Put $\breve{W}_{v}=\breve{j}_{v}\left(W_{v}\right)$ and $\check{\Sigma}_{v}=\check{j}_{v}(\Sigma)$. Let $F_{v}$ be a fundamental region for $\Gamma_{v}$ on $\Omega_{v}$ which contains $\check{W}_{v}$. Here we can assume that the $F_{v}$ are compact simplicial complexes embedded in $\Omega_{v}$, by considering triangulation of $X_{v}$. By $\check{j}_{v}^{-1}$, we regard $F_{v}$ as a subset in $\mathbf{P}^{3}$ which contains $W_{v}$, and $\check{\Sigma}_{v}$ as $\Sigma$. Put $F=\left(F_{1} \backslash W_{1}^{\prime}\right) \cup\left(F_{2} \backslash W_{2}^{\prime}\right)$ and $\Omega=\bigcup_{g \in \Gamma} g(F)$, where $\Gamma$ is the subgroup of PGL(4) generated by $\check{j}_{v}^{-1} \Gamma_{v} \check{j}_{v}, v=1,2$. Then we see that $X_{1} \# X_{2}$ is a compact quotient of $\Omega$ by $\Gamma$. Here $\Gamma$ is given by the free product $\left(\check{j}_{1}^{-1} \Gamma_{1} \breve{j}_{1}\right) *\left(\breve{j}_{2}^{-1} \check{\Gamma}_{2} \check{j}_{2}\right)$. In the following, we write $\Gamma_{v}$ instead of $\check{j}_{v}^{-1} \check{\Gamma}_{v} \check{j}_{v}$ for short. We put $\Lambda=\mathbf{P}^{3} \backslash \Omega$.

For a group $G$, we indicate the set $G \backslash\{1\}$ by $G^{*}$. Every element of $\Gamma^{*}$ can be written in the normal form

$$
g=g_{n} \circ \cdots \circ g_{1}
$$

where either $g_{2 i} \in \Gamma_{1}^{*}, g_{2 i+1} \in \Gamma_{2}^{*}$, or $g_{2 i} \in \Gamma_{2}^{*}, g_{2 i+1} \in \Gamma_{1}^{*}$. The number of factors $n$ in the right hand side of (15) is determined by the element $g$. We call $n$ the length of $g$, and denote the length by $|g|$. We set $|1|=0$. Furthermore, writing $g$ in the normal form (15), we say that $g$ is positive $(g>0)$ if $g_{1} \in \Gamma_{1}^{*}$, and negative $(g<0)$ if $g_{1} \in \Gamma_{2}^{*}$.

We decompose $\Gamma$ into the sets of positive elements, negative elements and the identity element, and write

$$
\Gamma=\{1\}+\sum_{n, \mu} p_{n \mu}+\sum_{n, \mu} q_{n \mu},
$$


where $\left|p_{n \mu}\right|=\left|q_{n \mu}\right|=n, p_{n \mu}>0$, and $q_{n \mu}<0$. For $n>0$, we set

$$
T_{n}=\bigcup_{\mu} p_{n \mu}\left(W_{1}\right) \cup \bigcup_{\mu} q_{n \mu}\left(W_{2}\right),
$$

and $T_{0}=W_{1} \cup W_{2}$.

LEMMA 5.1. For $n \geq 1, T_{n-1} \supset T_{n}$ holds.

Proof. Let $x \in T_{n}$ be any element. Suppose $x \in p_{n \mu}\left(W_{1}\right)$ for some $p_{n \mu}>0$. We write $p_{n \mu}=g_{n} \circ \cdots \circ g_{1}$ in the normal form, where $g_{1} \in \Gamma_{1}^{*}$. Since $g_{1}\left(W_{1}\right) \subset W_{2}$, we have

$$
p_{n \mu}\left(W_{1}\right) \subset g_{n} \circ \cdots \circ g_{2}\left(W_{2}\right) .
$$

Since $\left|g_{n} \circ \cdots \circ g_{2}\right|=n-1$ and $g_{n} \circ \cdots \circ g_{2}$ is negative, we conclude $x \in T_{n-1}$. We can settle the case $x \in q_{n \mu}\left(W_{2}\right)$ for some $q_{n \mu}<0$ in the same manner.

LEMMA 5.2. For any $g \in \Gamma$ with $|g| \leq n, n \geq 1, g\left(T_{n}\right) \subset T_{n-|g|}$ holds.

Proof. Take any $x \in T_{n}$. We write $g=g_{k} \circ \cdots \circ g_{1}$ in the normal form, $k=|g|$. Then we see easily that $g(x) \in \bigcup_{l=n-k}^{n+k} T_{l}$. Since $\left\{T_{n}\right\}$ is a descending sequence by Lemma 5.1, we have $g(x) \in T_{n-k}$.

We set

$$
T=\bigcap_{n \geq 1} T_{n}
$$

As a corollary to Lemma 5.2, we have immediately

LemmA 5.3. For any $g \in \Gamma, g(T) \subset T$ holds and hence $T$ is $\Gamma$-invariant.

LEMMA 5.4. For any $g \in \Gamma, T_{n} \cap g(F)=\emptyset$ holds for $n \geq|g|+1$.

Proof. It is clear by the definition that $T_{1} \cap F=\emptyset$. Since $\left\{T_{n}\right\}$ is a descending sequence by Lemma 5.1, we have

$$
T_{n} \cap F=\emptyset
$$

for $n \geq 1$. We shall prove the lemma by induction on $k=|g|$. The lemma holds for $k=0$ by (18). Suppose that $k \geq 1$. Put $g=g_{k} \circ h,|h|=k-1, g_{k} \in \Gamma_{v}^{*}$, where $v=1$ or 2 . Then we have

$$
\begin{aligned}
T_{n} \cap g(F) & =T_{n} \cap g_{k} \circ h(F) \\
& =g_{k}\left(g_{k}^{-1}\left(T_{n}\right) \cap h(F)\right) .
\end{aligned}
$$

For $n \geq 1$, we have

$$
g_{k}^{-1}\left(T_{n}\right) \subset T_{n-1}
$$

by Lemma 5.1. By the induction assumption, we have

$$
T_{n-1} \cap h(F)=\emptyset .
$$


Hence by (19), we have

$$
\begin{aligned}
T_{n} \cap g(F) & =g_{k}\left(g_{k}^{-1}\left(T_{n}\right) \cap h(F)\right) \\
& \subset g_{k}\left(T_{n-1} \cap h(F)\right) \\
& =\emptyset .
\end{aligned}
$$

LEMMA 5.5. $T \subset \Lambda$.

PROOF. We shall prove the lemma by contradiction. Suppose there were a point $x \in T$ which were contained in $\Omega=\mathbf{P}^{3} \backslash \Lambda$. Let $K$ be a compact neighborhood of $x$ in $\Omega$. By the definition of $T$ and the fact that $\left\{T_{n}\right\}$ is a descending sequence,

$$
x \in K \cap T_{n} \text { for all } n \geq 1 .
$$

Since $K$ and $F$ are compact, and since $F$ is a fundamental region, there is a finite number of elements $g_{1}, g_{2}, \cdots, g_{r} \in \Gamma$ such that

$$
K \subset \bigcup_{j=1}^{r} g_{j}(F)
$$

and

$$
K \cap g(F)=\emptyset \text { for all } g \in \Gamma \backslash\left\{g_{1}, g_{2}, \cdots, g_{r}\right\}
$$

Hence, by (20),

$$
\bigcup_{j=1}^{r} g_{j}(F) \cap T_{n} \supset K \cap T_{n} \supset\{x\} \neq \varnothing
$$

for all $n \geq 1$. On the other hand, by Lemma 5.4, we have $T_{n} \cap g_{j}(F)=\emptyset$ for all integers $n$ with

$$
n \geq \max _{j=1, \cdots, r}\left\{\left|g_{j}\right|+1\right\} .
$$

Thus we have a contradiction for such $n$.

We set

$$
S_{n}=\mathbf{P}^{3} \backslash T_{n}, \quad S=\bigcup_{n} S_{n}
$$

Then we have

LEMMA 5.6. $\Lambda=(\Lambda \cap S) \cup T$.

Proof. Since $\mathbf{P}^{3}=S \cup T$, the lemma follows immediately from Lemma 5.5.

Following three lemmas follow immediately from Lemmas 5.1, 5.2 and 5.3.

LEMMA 5.7. For $n \geq 1, S_{n-1} \subset S_{n}$. 
LEMMA 5.8. For any $g \in \Gamma$ and $n \geq 1, g\left(S_{n}\right) \supset S_{n-|g|}$ holds.

LEMMA 5.9. For any $g \in \Gamma, g(S) \subset S$ holds and hence $S$ is $\Gamma$-invariant.

Put

$$
L=\bigcup_{g \in \Gamma} g\left(\Lambda_{1}\right) \cup \bigcup_{h \in \Gamma} h\left(\Lambda_{2}\right)
$$

It is easy to verify the following.

LEMMA 5.10. If $x \in \mathbf{P}^{3} \backslash L$, then

$$
g(x) \in \Omega_{1} \cap \Omega_{2} \quad \text { for all } g \in \Gamma \text {. }
$$

LEMMA 5.11. $S \subset \Omega \cup L$.

Proof. Take $x \in S \cap\left(\mathbf{P}^{3} \backslash L\right)$. It is enough to show that $x \in \Omega$. Since $x \in S$ and $\left\{S_{n}\right\}_{n}$ is ascending, there is an integer $n_{0}$ such that $x \in S_{n_{0}} \backslash S_{n_{0}-1}$. Then $x \in T_{n_{0}-1}=$ $\mathbf{P}^{3} \backslash S_{n_{0}-1}$. Therefore either there is a point $w_{1} \in W_{1}$ and an element $p_{n_{0}-1 \mu}>0$ in $\Gamma$ with $x=p_{n_{0}-1 \mu}\left(w_{1}\right)$, or there is a point $w_{2} \in W_{2}$ and an element $q_{n_{0}-1 \mu}<0$ in $\Gamma$ with $x=q_{n_{0}-1 \mu}\left(w_{2}\right)$. Suppose the former case holds; the proof of the latter case is similar. If $w_{1} \in T_{1}$, then there is a point $v_{2} \in W_{2}$ and $q \in \Gamma_{2}^{*}$ such that $w_{1}=q\left(v_{2}\right)$. Hence we have $x=p_{n_{0}-1 \mu} \circ q\left(v_{2}\right)$. Since $p_{n_{0}-1 \mu} \circ q$ is negative and has length $n_{0}$, we see that $x \in T_{n_{0}}$. But this contradicts $x \in S_{n_{0}}$. Hence $w_{1} \notin T_{1}$, i.e., $w_{1} \in S_{1}$. Namely, for every element $x \in S_{n}$ with $n>1$, there is an element $g \in \Gamma$ such that $g(x) \in S_{1}$. On the other hand, by the choice of $x$ and by Lemma 5.10, we also have $g(x) \in \Omega_{1} \cap \Omega_{2}$. If $g(x) \in W_{2}$, then $g(x) \notin W_{1} \backslash W$. Since $g(x) \in \Omega_{1}, g_{1} \circ g(x) \in F_{1}$ holds for some $g_{1} \in \Gamma_{1}$. Suppose that $g_{1} \circ g(x) \in W_{1} \backslash W$. Then we have $g_{1} \neq 1$ and $g(x)=g_{1}^{-1}\left(g_{1} \circ g(x)\right) \in g_{1}^{-1}\left(W_{1} \backslash W\right) \subset T_{1}$. This contradicts $g(x) \in S_{1}$. Therefore $g_{1} \circ g(x) \in\left(F_{1} \backslash W_{1}^{\prime}\right) \cup W \subset F$. Hence $x \in \Omega$. The remaining case $g(x) \in W_{1}$ can be settled similarly.

LEMMA 5.12. $L \subset \Lambda$.

Proof. Since $\Lambda$ is $\Gamma$-invariant, it is enough to show that $\Omega \subset \Omega_{v}$. We consider the case $v=1$. The proof works also for $v=2$. Recall that $\Omega=\cup_{g \in \Gamma} g(F)$ and $\Omega_{1}=$ $\bigcup_{g \in \Gamma_{1}} g\left(F_{1}\right)$. Then $\Omega \subset \Omega_{1}$ follows immediately from the following

Sublemma 5.1. Let $g$ be an element of $\Gamma$ and put $k=|g|$. If $g$ is positive, then $g(F) \subset F_{2} \cap \Omega_{1}$ for odd $k$, and $g(F) \subset F_{1}$ for even $k$. If $g$ is negative, then $g(F) \subset F_{1}$ for odd $k$, and $g(F) \subset F_{2} \cap \Omega_{1}$ for even $k$.

Proof. Consider the case $g$ positive. We shall prove the sublemma by induction on $k$. If $k=1$, then $g \in \Gamma_{1}^{*}$. Hence $g(F) \subset g\left(F_{1}\right) \subset F_{2} \cap \Omega_{1}$. If $k=2$, then $g=g_{2} \circ g_{1}$ for some $g_{1} \in \Gamma_{1}^{*}$ and $g_{2} \in \Gamma_{2}^{*}$. Hence

$$
g(F) \subset g_{2} \circ g_{1}\left(F_{1}\right) \subset g_{2}\left(F_{2} \cap \Omega_{1}\right) \subset g_{2}\left(F_{2}\right) \subset F_{1} .
$$


Suppose that $k \geq 3$. Write $g$ as $g=g_{k} \circ h, h=g_{k-1} \circ \cdots \circ g_{1}$ If $k$ is odd, then $g_{k} \in \Gamma_{1}^{*}$ and $h(F) \subset F_{1}$ by induction assumption. Hence $g(F)=g_{k} \circ h(F) \subset g_{k}\left(F_{1}\right) \subset F_{2} \cap \Omega_{1}$. If $k$ is even, then $g_{k} \in \Gamma_{2}^{*}$ and $h(F) \subset F_{2} \cap \Omega_{1}$ by induction assumption. Hence

$$
g(F)=g_{k} \circ h(F) \subset g_{k}\left(F_{2} \cap \Omega_{1}\right) \subset g_{k}\left(F_{2}\right) \subset F_{1} .
$$

The case $g$ negative can be settled similarly.

Proposition 5.2 ([K5] Proposition 2.1). $\quad \Lambda=L \cup T$.

Proof. By Lemmas 5.6 and 5.11, we have $\Lambda \subset \Omega \cup L \cup T$. Hence, by $\Omega \cap \Lambda=\emptyset$, we obtain $\Lambda \subset L \cup T$. The other implication follows from Lemmas 5.12 and 5.5.

LEMMA 5.13. $L \cap T=\emptyset$.

Proof. Suppose that there is a point $x \in L \cap T$. Then by (21), there is $g \in \Gamma$ such that $x \in g\left(\Lambda_{1}\right)$ or $x \in g\left(\Lambda_{2}\right)$. We can assume that $x \in g\left(\Lambda_{1}\right)$ holds without loss of generality. Fix $n \in \mathbf{N}$ such that $n>\left|g^{-1}\right|+1$. Then, Since $x \in T \subset T_{n}$, there is either $p \in \Gamma$ such that $x \in p\left(W_{1}\right)$ with $|p|=n$ and $p>0$, or $q \in \Gamma$ such that $x \in q\left(W_{2}\right)$ with $|q|=n$ and $q<0$. Then either $x \in p\left(W_{1}\right) \cap g\left(\Lambda_{1}\right) \neq \emptyset$, or $x \in q\left(W_{2}\right) \cap g\left(\Lambda_{1}\right) \neq \emptyset$ holds. Therefore either $g^{-1} \circ p\left(W_{1}\right) \cap \Lambda_{1} \neq \emptyset$, or $g^{-1} \circ q\left(W_{2}\right) \cap \Lambda_{1} \neq \emptyset$ holds. Since $g^{-1} \circ p>0,\left|g^{-1} \circ p\right|>0$, $g^{-1} \circ q<0$, and $\left|g^{-1} \circ q\right|>0$, we see that $T_{1} \cap \Lambda_{1} \supset\left(g^{-1} \circ p\left(W_{1}\right) \cup g^{-1} \circ q\left(W_{2}\right)\right) \cap \Lambda_{1} \neq \emptyset$ holds. On the other hand, since $T_{1} \subset\left(\Omega_{1} \backslash W_{1}\right) \cup\left(\Omega_{2} \backslash W_{2}\right)$, we have $T_{1} \cap \Lambda_{1}=\emptyset$. Thus we have a contradiction.

LEMmA 5.14. For any $g, h \in \Gamma$ with $g \neq h$, we have $g\left(\Lambda_{1}\right) \cap h\left(\Lambda_{2}\right)=\emptyset$.

Proof. We can assume that $h=1$ and $g \neq 1$. Take any $g \in \Gamma^{*}$ and write it in the normal form $g=g_{n} \circ g_{n-1} \circ \cdots \circ g_{1}$. We shall show by induction on $|g|$ that if $g_{n}>0$ then $g\left(\Lambda_{1}\right) \subset W_{2}$ and that if $g_{n}<0$ then $g\left(\Lambda_{1}\right) \subset W_{1} \cap \Omega_{2}$. From the assertion, the lemma follows immediately. First suppose that $|g|=1$. If $g>0$, then $g\left(\Lambda_{1}\right)=\Lambda_{1} \subset W_{2}$. If $g<0$, then $g\left(\Lambda_{1}\right) \subset g\left(W_{2}\right) \subset W_{1} \cap \Omega_{2}$. Thus the case $|g|=1$ is settled. Next suppose that $|g|>1$. If $g_{n}>0$, then $g_{n-1}<0$. Hence $g_{n-1} \circ \cdots \circ g_{1}\left(\Lambda_{1}\right) \subset W_{1} \cap \Omega_{2}$ holds by the induction assumption. Therefore we have $g\left(\Lambda_{1}\right) \subset g_{n}\left(W_{1} \cap \Omega_{2}\right) \subset W_{2}$. If $g_{n}<0$, then $g_{n-1}>0$. Hence $g_{n-1} \circ \cdots \circ g_{1}\left(\Lambda_{1}\right) \subset W_{2}$ holds by the induction assumption. Therefore we have $g\left(\Lambda_{1}\right) \subset g_{n}\left(W_{2}\right) \subset W_{1} \cap \Omega_{2}$.

Let $\alpha$ be any connected component of $\Lambda$.

LEMMA 5.15. $\alpha \subset T$.

Proof. Take any point $x \in T$ and let $\alpha$ be the connected component of $\Lambda$ with $x \in \alpha$. Since $x \in T_{n}$ for any $n$, either $x \in g\left(W_{1}\right)$ with $g>0$ and $|g|=n$, or either $x \in h\left(W_{2}\right)$ with $h<0$ and $|h|=n$. If $x \in g\left(W_{1}\right)$ holds, then since $\alpha$ is connected and since $\alpha \cap g(\Sigma)=\emptyset, \alpha$ is contained in $g\left(W_{1}\right)$. Similarly, if $x \in h\left(W_{2}\right)$ holds, then $\alpha$ is contained in $h\left(W_{2}\right)$. Thus in any case we have $\alpha \subset T_{n}$. Therefore we have $\alpha \subset T$. 
Put

$$
\Omega_{v}^{\#}=\Omega_{v} \backslash \bigcup_{g \in \Gamma_{v}^{*}} g\left(W_{v}\right)=F_{v} \cup \bigcup_{g \in \Gamma_{v}^{*}} g\left(F_{v} \backslash W_{v}\right), \quad v=1,2 .
$$

Then we have $\Lambda \cap \Omega_{v}^{\#}=\emptyset$. Since either $\alpha \subset W_{1}$ or $\alpha \subset W_{2}$, we may assume here that $\alpha \subset W_{1}$. Since

$$
\alpha \cap \Omega_{2}^{\#} \subset \Lambda \cap \Omega_{2}^{\#}=\emptyset \quad \text { and } \quad \alpha \cap L=\emptyset,
$$

there is an element $g_{0} \in \Gamma_{2}^{*}$ such that $\alpha \subset g_{0}\left(W_{2}\right)$. Since

$$
g_{0}^{-1}(\alpha) \cap \Omega_{1}^{\#} \subset \Lambda \cap \Omega_{1}^{\#}=\emptyset \quad \text { and } \quad g_{0}^{-1}(\alpha) \cap L=\emptyset,
$$

there is an element $g_{1} \in \Gamma_{1}^{*}$ such that $g_{0}^{-1}(\alpha) \subset g_{1}\left(W_{1}\right)$. Since

$$
g_{1}^{-1} \circ g_{0}^{-1}(\alpha) \cap \Omega_{2}^{\#} \subset \Lambda \cap \Omega_{2}^{\#}=\emptyset \quad \text { and } \quad g_{1}^{-1} \circ g_{0}^{-1}(\alpha) \cap L=\emptyset \text {, }
$$

there is an element $g_{2} \in \Gamma_{2}^{*}$ such that $g_{1}^{-1} \circ g_{0}^{-1}(\alpha) \subset g_{2}\left(W_{2}\right)$. Continuing this process, we have a sequence $\left\{g_{n}\right\}_{n} \subset \Gamma$ with $g_{2 k-1} \in \Gamma_{1}^{*}, g_{2 k} \in \Gamma_{2}^{*}$ such that $\alpha \subset g_{0} \circ \cdots g_{2 k-1}\left(W_{1}\right)$. Here, we can also check in each step that $\alpha \subset g_{0} \circ \cdots \circ g_{2 k-1}\left(W_{1} \backslash W\right)$ hold. We put $p_{k}=g_{0} \circ \cdots \circ g_{2 k-1}$ and consider the sequence $\left\{p_{k}\right\}_{k=1}^{\infty}$. We put

$$
U_{k}=p_{k}\left(W_{1}\right), \quad K_{k}=p_{k}\left(W_{1} \backslash W\right), \quad \Sigma_{k}=p_{k}(\Sigma) .
$$

Then, we have for $k \geq 1$

$$
\alpha \subset K_{k+1} \subset U_{k+1} \subset K_{k} \subset U_{k} \subset W_{1},
$$

$$
\Sigma_{k} \subset U_{k} \backslash K_{k}
$$

By (24), we have

$$
K:=\bigcap_{k \geq 1} U_{k}=\bigcap_{k \geq 1} K_{k}
$$

is compact and

$$
\alpha \subset K
$$

In the following, we indicate by $\hat{M}$ the subset of $\operatorname{Gr}(4,2)$ which parametrizes the lines contained in a set $M \subset \mathbf{P}^{3}$. Each $p_{k}$ defines a biholomorphic map $\hat{p}_{k}$ of $\operatorname{Gr}(4,2)$ such that

$$
\hat{p}_{k}: \hat{W}_{1} \rightarrow \hat{U}_{k} \subset \hat{W}_{1} .
$$

The open subset $\hat{U}_{k} \subset \operatorname{Gr}(4,2)$ is biholomorphic to a domain of the form

$$
\left\{X \in M_{2}(\mathbf{C}): I-{ }^{t} \bar{X} X \text { is positive definite }\right\},
$$


which is a bounded Stein domain in $\mathbf{C}^{4}$ and $\hat{K}_{k}$ is a compact subset of $\hat{U}_{k}$, where $\hat{K}_{k}$ is of the form

$$
\left\{X \in M_{2}(\mathbf{C}): r I-{ }^{t} \bar{X} X \text { is positive semi-definite }\right\},
$$

for some constant $0<r<1$. Obviously we have $\hat{p}_{k}\left(\hat{U}_{k}\right)=\hat{U}_{k+1}$ and $\hat{p}_{k}\left(\hat{K}_{k}\right)=\hat{K}_{k+1}$. Therefore the holomorphic maps $\left\{\hat{p}_{k}\right\}_{k}$ constitutes a normal family on $W_{1}$. Let $\left\{\hat{q}_{k}\right\}_{k}$ be a uniformly convergent subsequence of $\left\{\hat{p}_{k}\right\}_{k}$ and put

$$
\hat{q}=\lim _{k \rightarrow \infty} \hat{q}_{k} .
$$

On the other hand, since $K_{k} \supset K_{k+1}$, we have easily

$$
\hat{K}=\bigcap_{k=1}^{\infty} \hat{K}_{k} .
$$

LEMMA 5.16. $\hat{q}: \hat{W}_{1} \rightarrow \hat{K}$ is surjective.

Proof. Take any point $a \in \hat{K}$. Then there is a line $\ell_{a} \subset K$. Since, $\ell_{a} \subset K_{k}$ for any $k$, we can consider a line $\ell_{k}=q_{k}^{-1}\left(\ell_{a}\right)$ in $W_{1} \backslash W$. Choosing a subsequence of $\left\{\ell_{k}\right\}_{k}$, we can assume that $\lim _{k} \hat{\ell}_{k}=\hat{\ell}_{0}$ for some $\hat{\ell}_{0}$ in $\left(W_{1} \backslash W\right)^{\wedge}$. This is possible because $\left(W_{1} \backslash W\right)^{\wedge}$ is compact. Since the convergence of $\left\{\hat{q}_{k}\right\}_{k}$ is uniform on $\left(W_{1} \backslash W\right)^{\wedge}$, we have $\hat{q}\left(\hat{\ell}_{0}\right)=\lim _{k} \hat{q}_{k}\left(\ell_{0}\right)=\lim _{k} \hat{q}_{k}\left(\hat{\ell}_{k}\right)=\ell_{a}$.

LEMMA 5.17. $\hat{K}$ consists of a single point.

Proof. Let $f$ be any holomorphic function on $\hat{W}_{1}$. Then $|f|_{\hat{K}}$ has a maximum point on $\hat{K}$. By Lemma 5.16, $|f \circ \hat{q}|$ has a maximum point on $\hat{W}_{1}$. Thus the holomorphic function $f \circ \hat{q}$ is constant on $\hat{W}_{1}$. Since $\hat{W}_{1}$ is of Stein, this implies the lemma.

LEMmA 5.18. K coincides with the support of a line.

Proof. Through every point of $K$, there passes a line in $K$. Thus we have the lemma from Lemma 5.17.

Lemma 5.19. Any connected component $\alpha$ of $T$ coincides with a line.

Proof. Since $\alpha$ contains at least a line, the lemma follows from (26) and Lemma 5.18 .

ProOf OF Proposition 5.1. The proposition is immediate from Lemmas 5.13, 5.14, 5.19, and Proposition 5.2.

Notes ADDED IN PRoOF. The example 1 given in [K3] as a Blanchard manifold of type (A) was not correct, since Lemma A.6 in the appendix of [K3] was false. We can disprove the existence of Blanchard manifolds of type (A). This will be published elsewhere. 


\section{References}

[D] Dloussky, G.: Envelopes d'holomorphie et prolongements d'hypersurfaces, Seminaire Pierre Lelong p. 215-235, Année 1975-76, Lec. Notes in Math. 578, Springer (1977).

[F] FUJIKI, A.: Topology of compact self-dual manifolds whose twistor space is positive algebraic dimension, J. Math. Soc. Japan 54 (2002), 587-608.

[G] Gauduchon, P.: Le théorème de l'excentricité nulee, C. R. Acad. Sc. Paris, t. 285 (1977), Série A 387-390.

[I] IVAShKovich, S. M.: Extension properties of meromorphic mappings with values in non-Kähler complex manifolds, Ann. of Math. 160 (2004), 795-837.

[K1] Kato, MA.: Complex structures on $S^{1} \times S^{5}$, Jour. Math. Soc. of Japan, 28 (1976), 550-576.

[K2] KATO, MA.: On compact complex 3-folds with lines, Japanese J. Math. 11 (1985), 1-58.

[K3] KATO, MA.: Factorization of compact complex 3-folds which admit certain projective structures, Tohoku Math. J. 41 (1989), 359-397.

[K4] KATO, MA.: Examples on an extension problem of holomorphic maps and a holomorphic 1-dimensional foliation, Tokyo J. Math. 13 (1990), 139-146.

[K5] Kato, MA.: Compact quotient manifolds of domains in a complex 3-dimensional projective space and the Lebesgue measure of limit sets, Tokyo J. Math. 19 (1996), 99-119.

[KO] KAтO, MA.; Okada, N.: On holomorphic maps into compact non-Kähler manifolds, Ann. Inst. Fourier, Grenoble 54 (2004), 1827-1854.

[L] LÁRUSSON, F.: Compact quotients of large domains in complex projective space, Ann. Inst. Fourier, Grenoble 48, 1 (1998), 223-246.

[M] Maskit, B.: On Klein's combination theorem III, Ann. Math. Studies 66 (1971) 297-316.

[S] SIU, Y. T.: Techniques of extension of analytic objects, Dekker, New York (1974).

[U] UEno, K.: Classification theory of algebraic varieties and compact complex spaces, Lec. Notes Math. 439, Springer-Verlag (1975).

Present Address:

DEPARTMENT OF MATHEMATICS, SOPHIA UNIVERSiTy,

KIOICHO 7-1, TOKYO, 102-8554 JAPAN.

e-mail:kato@mm.sophia.ac.jp 